\title{
Analysis of drug sensitivity of human high-grade osteosarcoma in a chick chorioallantoic membrane (CAM) model: a proof of principle study
}

\author{
Wiebke K. Guder ${ }^{1,2^{*}}$ (D), Wolfgang Hartmann ${ }^{3}$, Marcel Trautmann ${ }^{3}$, Jendrik Hardes ${ }^{1,2}$, Eva Wardelmann ${ }^{3}$,
} Maurice Balke $e^{4}$ and Arne Streitbürger ${ }^{1,2}$

\begin{abstract}
Objective: Multi-agent chemotherapy is an important pillar in treatment of high-grade osteosarcoma. In an effort to improve patient survival, it is imperative to determine the effectiveness of new substances. The objective of this study was to investigate whether the chick chorioallantoic membrane (CAM) model can be used to analyze drug sensitivity in high-grade osteosarcoma.

Results: Spare biopsy tissue from five patients diagnosed with high-grade osteosarcoma was transferred into nonimmortalized primary cell culture. After a pre-incubation period of 10 days, fertilized chick eggs were inoculated with primary tumor cells suspended in extracellular matrix gel. On day 16, treatment with $20 \mu \mathrm{mol} / \mathrm{l}$ doxorubicin $(n=4)$ or $25 \mu$ l of culture medium $(n=6)$ was performed for 24 h. CAM membranes were documented macroscopically, harvested and examined histologically. Transfer of biopsy specimens into primary cell culture was successful in all cases. $50 \%(n=10)$ of eggs died after inoculation with tumor cells and before application of doxorubicin. No deaths occurred after application of doxorubicin. Histological examination found a response to doxorubicin in all four specimens. Based upon these results, the CAM model represents a promising preclinical alternative to animal experiments to determine drug sensitivity of osteosarcoma cells. Further research with regard to other substances and dosages appear justified.
\end{abstract}

Keywords: Chorioallantoic membrane model, CAM, Osteosarcoma, Primary cell culture

\section{Background}

High-grade osteosarcoma is a rare disease, mostly affecting children, adolescents and young adults. A combination of chemotherapy and wide tumor resection are the main pillars for successful treatment of these patients. The significance of chemotherapy

*Correspondence: wiebke.guder@uk-essen.de

1 Department of General and Tumor Orthopedics, University Hospital

Muenster, Albert-Schweitzer-Campus 1, Building A1, 48149 Münster,

Germany

Full list of author information is available at the end of the article becomes clear when considering how overall survival rates improved from $20 \%$ to more than $60 \%$ with the introduction of multi-agent chemotherapy [1]. Standard chemotherapeutic agents include doxorubicin, cisplatin, ifosfamide and methotrexate [2]. Patients are usually enrolled in multimodal clinical trials in an effort to improve treatment outcomes by comparing new approaches to a standard regimen. Current protocols intend patients to undergo neoadjuvant chemotherapy to evaluate response to chemotherapy as the percentage of tumor regression found in the resection specimen of the primary tumor. As a result, good

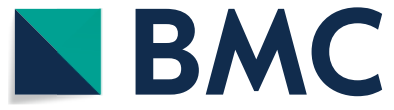

(c) The Author(s) 2020. This article is licensed under a Creative Commons Attribution 4.0 International License, which permits use, sharing, adaptation, distribution and reproduction in any medium or format, as long as you give appropriate credit to the original author(s) and the source, provide a link to the Creative Commons licence, and indicate if changes were made. The images or other third party material in this article are included in the article's Creative Commons licence, unless indicated otherwise in a credit line to the material. If material is not included in the article's Creative Commons licence and your intended use is not permitted by statutory regulation or exceeds the permitted use, you will need to obtain permission directly from the copyright holder. To view a copy of this licence, visit http://creativecommons.org/licenses/by/4.0/. The Creative Commons Public Domain Dedication waiver (http://creativecommons.org/publicdomain/zero/1.0/) applies to the data made available in this article, unless otherwise stated in a credit line to the data. 
and poor responders are assigned to different adjuvant chemotherapy groups, which vary with regard to duration and composition of remaining treatments [2]. However, evidence regarding the efficacy of this risk-stratified treatment approach is still lacking [3, 4]. Therefore, in case of poor response to chemotherapy, an antedated operation or modulation of administered chemotherapy might be desirable. However, no significant developments have been made with regard to new drugs in osteosarcoma protocols over the last few decades which may also be due to the lack of suitable preclinical individualized models having the power to reliably predict response $[1,3,4]$.

Preclinical research currently relies on in vitro and mouse models for drug screens to identify promising substances that might be translated into a clinical trial [5]. The latter in vivo xenograft models tend to be lengthy and unable to yield short-term information that might be able to influence individual treatment approaches. Beyond that, there has been a paradigm shift in our scientific society: ethical recommendations challenge scientists to develop models that do not rely on animal experiments whenever possible. In this context, the chorioallantoic membrane (CAM) model is particularly interesting as the chick embryo is not considered to be a living animal until day 17 of development in most countries and therefore does not fall under animal experiment $[6,7]$. Though successful engraftment of musculoskeletal tumors including human osteosarcoma cell lines has been reported and though there is some preliminary evidence that it may serve as a preclinical screening assay predictive for disease outcome, it is not in common use yet $[8,9]$.

Therefore, it is the objective of this study to analyze whether the CAM model may serve as a reliable preclinical drug-screening assay and if so, may even be predictive of response of the tumor to chemotherapy seen in the resected specimen of the primary tumor in a follow-up study.

\section{Main text \\ Material and methods Biopsy aliquots}

Biopsy aliquots used in this study were obtained from five patients who underwent diagnostic incisional biopsy for suspected high-grade osteosarcoma at our clinic. Pathologists separated a biopsy aliquot from the existing tissue provided that sufficient tumor tissue was available without impairing standard diagnostic procedures. Histological examination confirmed highgrade osteosarcoma in all five cases.

\section{Aliquot preparation}

Biopsy aliquots were minced in a petri dish using a scalpel under laminar airflow. 1-2 ml DMEM was added and the biopsy tissue strained through a sieve (Corning ${ }^{\circledR}$ Costar $^{\circledR}$ Cell Strainer $\left.100 \mu \mathrm{m}\right)$. The suspension was centrifuged for $3 \mathrm{~min}$ at 800 revolutions per minute (RPM). Then, the supernatant was pipetted into a T75 tissue culture flask. The remaining pellet was digested for $15 \mathrm{~min}$ at $5 \% \mathrm{CO}_{2}$ and $37^{\circ}$ Celsius using 5-7 ml trypsin (trypsin EDTA, 0.25\%). Tissue digestion was inhibited adding 5-7 $\mathrm{ml} \mathrm{DMEM}$, and the suspension was centrifuged again as mentioned above. The supernatant was removed, the pellet solved in DMEM and then transferred into a T25 tissue culture flask.

\section{Primary cell culture}

All primary cell lines were grown in DMEM (Gibco ${ }^{\mathrm{TM}}$ Dulbecco's Modified Eagle Medium, high glucose, GlutaMAX ${ }^{\mathrm{TM}}$ ) supplemented with $10 \%$ fetal bovine serum (FBS), 1\% L-glutamine and 1\% penicillin/streptomycin on T25, T75 or T175 tissue culture flasks in a humidified atmosphere of $5 \% \mathrm{CO}_{2}$ at $37^{\circ}$ Celsius. Tissue culture flasks were coated in poly-L-lysine and washed with DPBS (Gibco ${ }^{\mathrm{TM}}$ Dulbecco's phosphate-buffered saline) in preparation of tumor-derived single cell suspensions. During incubation, vitality of primary cell cultures was checked on a daily basis under a light microscope. Cell culture medium was exchanged and the cell lines expanded depending on cell adherence, cell count and quality of culture medium.

\section{Chorioallantoic membrane (CAM) model}

CAM protocols as proposed by Sys et al. and Balke et al. were adapted to our study design $[10,11]$. Fertilized, pathogen-free eggs $(n=88)$ were purchased from and delivered by VALO BioMedia GmbH (OsterholzScharmbeck, Germany). These were kept in a wine cooler at $12-14^{\circ}$ Celsius and a humidity of $70-80 \%$ as shortly as possible. After transfer into the incubator, egg development was counted in days and eggs were exclusively handled under laminar airflow when outside the incubator. Incubation was performed in a sideways position at $37.5^{\circ}-38^{\circ}$ Celsius and a humidity of $70-80 \%$. On day 3 of egg development, a 1-2 mm hole was poked into the shallow pole of the egg, and $3 \mathrm{ml}$ of egg white was withdrawn from the egg to separate the CAM membrane from the eggshell. The hole was then sealed using adhesive tape. Subsequently, a circular window of approximately $2 \mathrm{~cm}$ in diameter was cut into the eggshell. The egg white was then returned into the egg and the circular window was sealed by adhesive tape. Eggs without an embryo were removed from the experiment. Egg vitality was checked 
on a daily basis and devitalized eggs were removed from the incubator. On day 10 of egg development, cells were harvested from primary cell culture and suspended at $10^{6}$ cells $/ 100 \mu \mathrm{l}$ in extracellular matrix gel (ECM). Per egg $(\mathrm{n}=20), 100 \mu \mathrm{l}$ of this suspension were topically applied in layers on the CAM membrane after careful superficial incision performed with a scalpel to facilitate ingrowth of the tumor on the CAM. Daily check-ups of egg vitality and removal of dead eggs was continued. On day 16 of egg development, remaining eggs $(n=10)$ were randomly divided into a treatment and a control group and either topically applied with $25 \mu \mathrm{l}$ of $20 \mu \mathrm{mol} / \mathrm{l}$ doxorubicin $(n=4)$ or $25 \mu$ l cell culture medium $(n=6)$. After $24 \mathrm{~h}$, on day 17 of egg development, the CAMs were covered in formaldehyde solution for $30 \mathrm{~min}$ before the area of interest (macroscopic tumor growth) was excised and processed for histological examination.

\section{Histological analysis}

Tissue workup followed standard routines including fixation of the tumors in $3.7 \%$ buffered formalin for $24 \mathrm{~h}$, standard dehydration and paraffin embedding followed by the preparation of paraffin blocks. Hematoxylin and eosin (H\&E) and Verhoeff's van Gieson (EVG) staining were performed on $3 \mu \mathrm{M}$ tissue sections following standard protocols.

\section{Results}

\section{Primary cell culture}

Transfer of processed biopsy aliquots into non-immortalized primary cell cultures including in vitro expansion of these tumor-derived single cell suspensions was successful in all specimens $(n=5)$.

\section{Chorioallantoic membrane (CAM) model}

Overall, egg survival was $86.3 \%(n=76 / 88)$ at day 3 , $54.5 \%(\mathrm{n}=48 / 88)$ at day 10 and $37.5 \%(\mathrm{n}=33 / 88)$ on day 17 of egg development. $50 \%$ of eggs $(n=10)$ inoculated with tumor cells on day $10(n=20)$ died before application of doxorubicin on day 16 . All remaining eggs $(n=10)$ survived until termination of the experiment $24 \mathrm{~h}$ after application of doxorubicin.

\section{Histological analysis}

Viable, CAM infiltrating tumors recapitulating osteosarcoma tumors with extensive osteoid formation were found in all specimen gained from the control and treatment group with the latter showing partial necrosis and
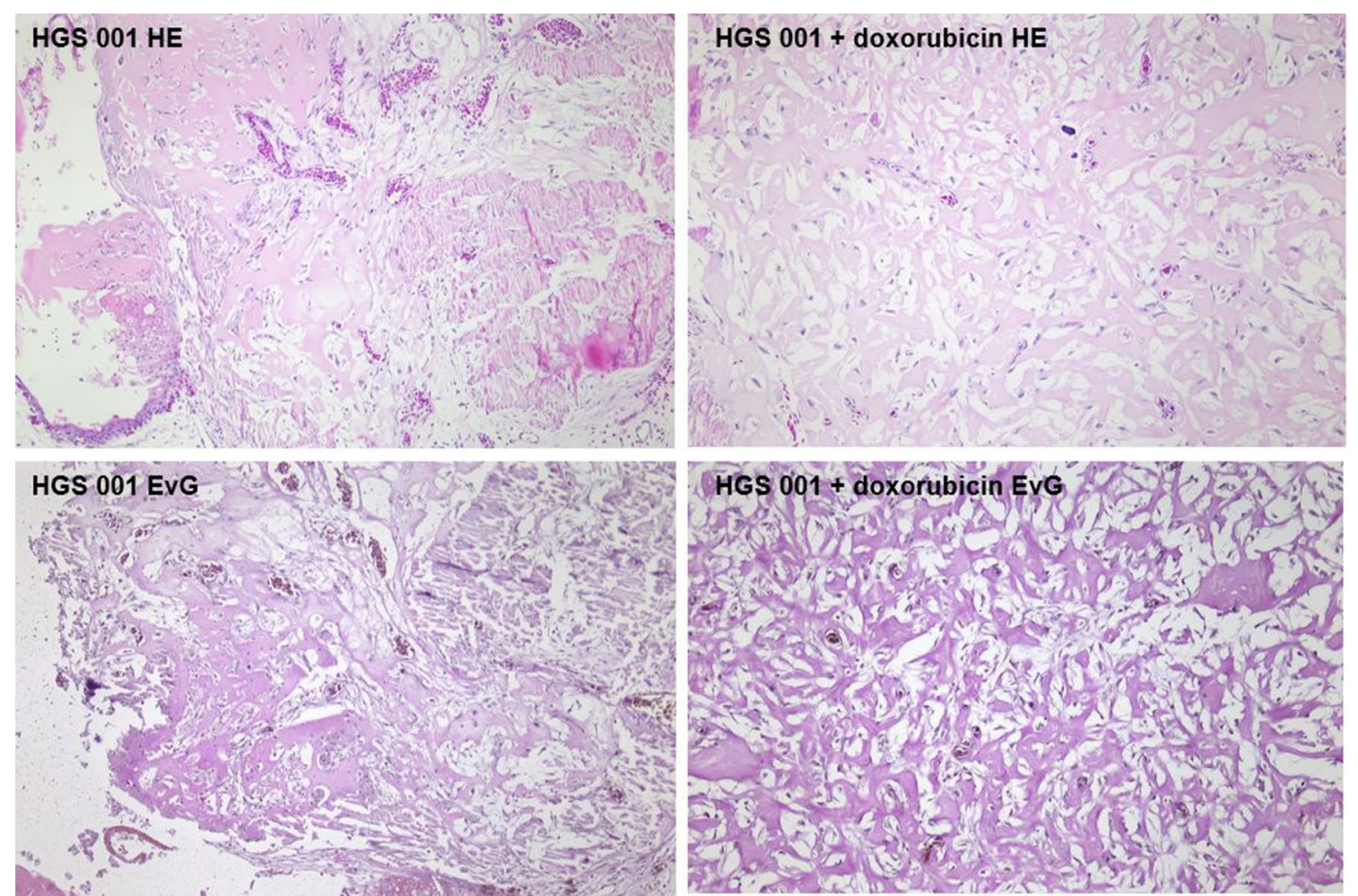

Fig. $1 \mathrm{HE}$ and EVG staining of tumors grown from the same primary cell culture (HGS001) without (left side) and after (right side) application of doxorubicin 

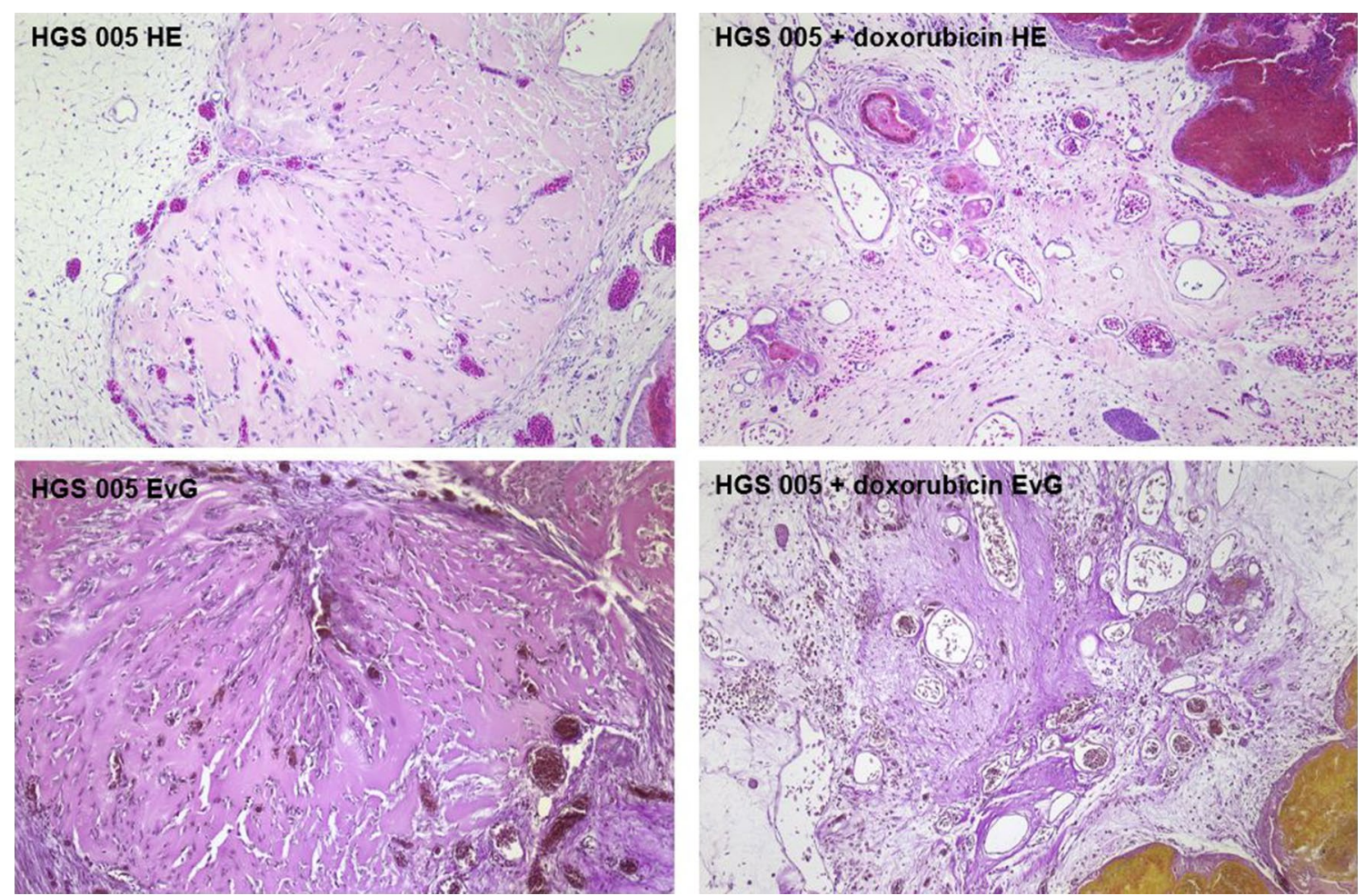

Fig. $2 \mathrm{HE}$ and EVG staining of tumors grown from the same primary cell culture (HGS005) without (left side) and after (right side) application of doxorubicin

hemorrhage as correlates of tumor regression while osteoid formation was retained (Figs. 1 and 2).

\section{Discussion}

In this preliminary study investigating the aptitude of the CAM model in testing drug sensitivity of high-grade osteosarcoma, we were able to show a therapeutic effect of doxorubicin on high-grade osteosarcoma grown on the CAM from tumor-derived single cell suspensions after prior expansion in non-immortalized primary cell culture.

While Sys et al. and others have reported the CAM to be a suitable model to grow musculoskeletal tumors and have suggested it as a model to test drug sensitivity, to our knowledge this study is the first one to prove a therapeutic effect of a chemotherapeutic agent on a tumor grown from primary osteosarcoma cells in the CAM $[8$, 9]. The results presented in our study, such as a high rate of embryo deaths throughout the incubation process, are in accordance with other reports in literature $[8,9]$ with Sys et al. reporting a rate of $20 \%$ of embryo deaths during the incubation period in their 2012 study [8]. Despite the fact that roughly two-thirds of the egg population died in the process of incubation and after inoculation with tumor cells, we still believe the CAM model to be a prospective alternative to other established mammalian models. It is a short-period, low-cost model from which conclusions can still be drawn from an adequate number of surviving eggs by repetition of the same protocol if need be. Further interest in this model may be drawn from the fact that it is not considered an animal experiment and therefore is in accordance with endeavors to avoid the employment of animals in preclinical research. The only alternative to date, which might manage without animal experiments altogether are bioengineered systems trying to mimic key aspects of human tumor microenvironments. Studies, such as an investigation by Marturano-Kruik et al. examining the biomechanical regulation of drug sensitivity in an engineered model of human tumor need to be compared with the results gained in CAM experiments to determine each model's aptitude in the future [12].

Therefore, follow-up studies are necessary to assess the significance this proof of principle has on future research projects. Particularly, the representation and comparability of tumor cell clones expanded in cell culture compared to the entire tumor needs to be verified. Also, other chemotherapeutic agents and their impact on tumor growth on the CAM need to be evaluated. Dose dependency of substance response for each substance 
has to be analyzed and compared with or translated into a clinical setting. Furthermore, additive effects caused by a combination of chemotherapeutic agents will have to be observed and compared with the CAM, other models and clinical experiences. Assessment on whether response to chemotherapy can be classified by grade of tumor necrosis and whether results compare with clinical results are necessary as tumor specimens grown on the CAM are small compared to the primary tumors they are supposed to represent. And lastly, it remains to be seen whether the human and CAM environment are similar enough to yield the same results. For example, the egg embryo's delayed development of an immune system [6] might affect tumor growth and response to chemotherapy and produce results that might be different in a human.

\section{Conclusion}

We believe the results presented in this study prove that analysis of drug sensitivity is possible on the CAM and follow-up studies investigating this model's clinical applicability are justified.

\section{Limitations}

Due to a very small number of evaluated eggs $(n=10)$ in this preliminary proof of principle study, investigation of tumor volumes with and without application of a chemotherapeutic agent did not yield meaningful results yet and further assessment will be necessary in larger followup studies.

\section{Abbreviations \\ CAM: Chorio-allantoic membrane; DMEM: Dulbecco's Modified Eagle Medium; DPBS: Dulbecco's phosphate-buffered saline; ECM: Extracellular matrix; FBS: Fetal bovine serum; RPM: Revolutions per minute. \\ Acknowledgements \\ Special thanks go to Esther-Pia Jansen and the staff at the Gerhard Domagk Institute of Pathology for their support in processing the CAM specimen for histological examination.}

\section{Authors' contributions}

WKG made substantial contributions to conception and design of the study, conducted the study, collected, analyzed and interpreted the acquired data and drafted the manuscript. WH made contributions to study design, performed the histological analysis of existing samples, was involved in drafting the manuscript and revised the manuscript critically for intellectual content. MT made contributions to study design (primary cell culture) and conduction of the study. JH revised the manuscript critically for intellectual content. EW revised the manuscript critically for intellectual content from a pathologist's perspective. MB made contributions to study design (CAM assay) and conduction of the study. AS made substantial contributions to conception and design of the study and revised the manuscript critically for intellectual content. All authors read and approved the final manuscript.

\section{Funding}

Open access funding provided by Projekt DEAL. This research did not receive any specific grant from funding agencies in the public, commercial or not-forprofit sectors.

\section{Availability of data and materials}

The datasets used and/or analyzed during the current study are available from the corresponding author on reasonable request.

\section{Ethics approval and consent to participate}

All procedures involving human participants were in accordance with the ethical standards of the institutional and/or national research committee and with the 1964 Helsinki declaration and its later amendments or comparable ethical standards. All procedures performed in research involving animals were in accordance with the ethical standards of Directive 2010/63/EU. Formal consent from the local Ethics Committee was obtained (Ethical committee of the Medical Council Westfalen-Lippe and Westphalian Wilhelms-University Muenster, Germany; Ref. number 2016-485-f-S).All patients whose tissues were included in this study gave written consent to participation in this study and publication of findings in an anonymous fashion.

\section{Consent for publication}

Not applicable.

\section{Competing interests}

The authors declare that they have no competing interests.

\section{Author details}

${ }^{1}$ Department of General and Tumor Orthopedics, University Hospital Muenster, Albert-Schweitzer-Campus 1, Building A1, 48149 Münster, Germany.

${ }^{2}$ Division of Musculoskeletal Oncology, University Hospital Essen, Hufelandstrasse 55, 45147 Essen, Germany. ${ }^{3}$ Gerhard Domagk Institute of Pathology, University Hospital Muenster, Albert-Schweitzer-Campus 1, Building D17, 48149 Münster, Germany. ${ }^{4}$ Sportsclinic Cologne, Ostmerheimer Strasse 200, 51109 Cologne, Germany.

Received: 18 June 2020 Accepted: 4 September 2020

Published online: 15 September 2020

\section{References}

1. Winkler K, Beron G, Delling G, et al. Neoadjuvant chemotherapy of osteosarcoma: results of a randomized cooperative trial (COSS-82) with salvage chemotherapy based on histological tumor response. J Clin Oncol. 1988;6(2):329-37.

2. Whelan JS, Bielack SS, Marina N, et al. EURAMOS-1, an international randomised study for osteosarcoma: results from pre-randomisation treatment. Ann Oncol. 2015;26(2):407-14. https://doi.org/10.1093/annon c/mdu526.

3. Bielack SS, Smeland S, Whelan JS, et al. Methotrexate, doxorubicin, and cisplatin (MAP) plus maintenance pegylated interferon alfa- $2 \mathrm{~b}$ versus MAP alone in patients with resectable high-grade osteosarcoma and good histologic response to preoperative MAP: first results oft he EURAMOS-1 good response randomized controlled trial. J Clin Oncol. 2015;33(20):2279-87. https://doi.org/10.1200/JCO.2014.60.0734.

4. Marina NM, Smeland S, Bielack SS, et al. Comparison of MAPIE versus MAP in patients with a poor response to preoperative chemotherapy for newly diagnosed high-grade osteosarcoma (EURAMOS-1): an open-label, international, randomised controlled trial. Lancet Oncol. 2016;17(10):1396408. https://doi.org/10.1016/S1470-2045(16)30214-5.

5. Bandyopadhyay A, Favours E, Phelps DA, et al. Evaluation of patritumab with or without erlotinib in combination with standard cytotoxic agents against pediatric sarcoma xenograft models. Pediatr Blood Cancer. 2018. https://doi.org/10.1002/pbc.26870.

6. Ribatti D. The chick chorioallantoic membrane as a model for tumor biology. Exp Cell Res. 2014;328(2):314-24. https://doi.org/10.1016/j.yexcr .2014.06.010.

7. Ribatti D. The chick embryo chorioallantoic membrane (CAM). A multifaceted experimental model. Mech Dev. 2016;141:70-7. https://doi. org/10.1016/j.mod.2016.05.003.

8. Sys $G$, Van Bockstal M, Forsyth $R$, et al. Tumour grafts derived from sarcoma patients retain tumour morphology, viability, and invasion potential and indicate disease outcomes in the chick chorioallantoic 
membrane model. Cancer Lett. 2012;326(1):69-78. https://doi. org/10.1016/j.canlet.2012.07.023.

9. Balke M, Neumann A, Kersting C, et al. Morphologic characterization of osteosarcoma growth on the chick chorioallantoic membrane. BMC Res Notes. 2010;3:58. https://doi.org/10.1186/1756-0500-3-58.

10. Sys GM, Lapeire L, Stevens N, et al. The in ovo CAM-assay as a xenograft model for sarcoma. J Vis Exp. 2013;77:e50522. https://doi. org/10.3791/50522.

11. Balke M, Neumann A, Szuhai K, et al. A short-term in vivo model for giant cell tumor of bone. BMC Cancer. 2011;11:241. https://doi. org/10.1186/1471-2407-11-241.
12. Marturano-Kruik A, Vilasante A, Yaeger K, et al. Biomechanical regulation of drug sensitivity in an engineered model of human tumor. Biomaterials. 2018;150:150-61. https://doi.org/10.1016/j.biomaterials.2017.10.020.

\section{Publisher's Note}

Springer Nature remains neutral with regard to jurisdictional claims in published maps and institutional affiliations.
Ready to submit your research? Choose BMC and benefit from:

- fast, convenient online submission

- thorough peer review by experienced researchers in your field

- rapid publication on acceptance

- support for research data, including large and complex data types

- gold Open Access which fosters wider collaboration and increased citations

- maximum visibility for your research: over $100 \mathrm{M}$ website views per year

At BMC, research is always in progress.

Learn more biomedcentral.com/submissions 